\title{
Exploration and Prospect of 3D Virtual Fitting Technology
}

\author{
Hongliang Xing \\ Shenyang Aerospace University
}

Keywords: 3D virtual fitting, shopping online, development prospect.

\begin{abstract}
The invention of 3D virtual fitting technology not only reflects the extension of fitting room in traditional shopping mall, but also breaks the restriction of reality, so users can have richer virtual fitting experience and the technology widens consumers' selection on clothes. The development of 3D virtual fitting technology makes construction need and practical operation of 3D online store possible, and it reflects favorable development prospect.
\end{abstract}

\section{Introduction}

With the rapid development of network technology and higher pace of life, buying clothes online has become one of the important transactions of shopping online. However, buying clothes online is not perfect. Consumers fail to fit on like in real stores and fail to see what do they look like when they fit on new clothes. In order to solve the problem that consumers cannot fit on clothes in shopping online, virtual fitting emerges as the times require. Virtual fitting technology provides lifelike online fitting environment and brand new shopping experience for consumers to assure that consumers can select satisfied clothes online. Therefore, the development of virtual fitting technology becomes can important topic in digital clothing technology field study and reflects diversified and practical development trends.

\section{Overview of $3 D$ virtual fitting technology}

3D virtual fitting technology refers to the technology of building customer's physical model with the help of computer figure and real-time video collection by using 3D visual principle. Fitting system processes images according to user's BWH information and "put" the clothes that customer select online on the customer to generate 3D fitting effect figure of model automatically ${ }^{[1]}$ and to create 3D virtual fitting model. 3D anthropometric technology is divided into contact type and non-contact type. Contact 3D anthropometric technology uses probe to sense the surface of the tested, which is used to measure human body parameters but is not suitable to measure real man. Non-contact 3D anthropometric technology is the major way of human body measurement. Light-sensitive equipment is used to capture the lights projecting on human body to generate images, which are used to describe human body's 3D features and can acquire human body's feature data rapidly, accurately, and efficiently. The generally used measuring methods include laser measuring method and infrared ray scan method.

3D virtual fitting technology can make customers feel whether clothes are suitable and can be satisfied mentally. With 3D virtual fitting system, people can see dressing effect before producing clothes, which not only helps dress designers concept and design but also helps customers make the best selection ${ }^{[2]}$. Meanwhile, the technology solves the problem that consumers cannot select details in shopping online; satisfies consumers' new needs; provides a brand new platform for dressing selection for the public. With the technology, shopping becomes more humanized.

\section{3D virtual fitting technology structure}

3D virtual fitting system technology is to integrate modern multidisciplinary technology to process relevant image information, such as optical electronics, computer graphics, information processing, computer visual and sensing technology and so on. When measuring human body, it 
regards the images of the tested as the carrier of testing or conveying information to use ${ }^{[3]}$. With the help of control points of human body features, the changeable parameterized 3D human body model can be built. Users can input data of height, waistline, shoulder breadth, chest width, and leg length and select built-in face shape, hairstyle, and skin color. The system extracts useful information according to human body feature data to build a model in the same scale of real man with the help of 3D human body modeling technology. The system is fast, accurate, efficient and can acquire mass data.

Virtual fitting operation includes selection of pattern, type, color, and fabric. First, the system ranks garment pieces around the human model according to the position of model to confirm the sewing relations to sew virtually. Then, the system sews garment pieces virtually. After virtual model fits on the virtual dressing, the system will show the fitting effect for people to observe the whole satisfaction, including virtual pendulous effect, fabric texture and texture effect, pattern and color effect. Furthermore, virtual dressing should not only reflect static simulation effect, but should also reflect dynamic simulation effect. Only in this way, can people observe the fitting effect from different perspectives.

\section{Advantages of 3D virtual fitting technology}

\subsection{More reasonable fitting experience}

Virtual fitting is a freer experience space and more comfortable interactive shopping environment, which can be regarded as the supplement and strength of traditional shopping online. Virtual fitting system not only can satisfy users' virtual experience, help customers cut the dress according to their figures to some degree, reflect the matching between dressing and users' characteristics more directly, but also can make customers see matching effect, acquire the same feeling with practical fitting, bring convenience for users to select the dress that they like, realize their richer virtual experience, and stimulate users' purchasing desire.

\subsection{More vivid fitting effect}

3D virtual fitting technology adopts scanner to measure body for customers, which avoids manual operation, reduces error, improves accuracy. It can provide the most accurate effect for customers. 3D virtual fitting not only includes the matching of model and color of dressing, but also can show the pendulous effect of fabric, lustre and change of movement. The fitting effect will be more real. Users will not worry about whether the size of dressing is suitable. Users will not worry about getting a refund or exchange. The technology is helpful for users to make better choice and is helpful to raise the success rate of dressing purchase.

\subsection{Shorter time for fitting}

There is huge data supporting system in 3D virtual fitting backstage, so consumers can "touch" fabric online and can change "new dressing" within a short time, which is very convenient and fast. People can choose any clothes that they like online. It is not necessary for them to go to shopping mall to fit on new clothes, which saves a lot of time and energy.

\subsection{Wider fitting view}

With virtual fitting system, people not only can see different effects when they put on different clothes directly and conveniently, but also can modify it to the ideal state as they like to assure that consumers can choose suitable dressing. The system can also make up the weaknesses of single observation angle, weak interaction, and separation of information and object of clothing sales online. Users can be satisfied mentally and users will have more selections.

\section{Prospect of 3D virtual fitting technology}

3D virtual fitting room appeared in America, Germany, and Japan for the first time. Now, some foreign products have been fitted on, matched, designed, and revised with 3D virtual fitting technology already. The technology can reflect the animation effect whether customers are comfortable when putting on clothes, simulate 3D pendulous effects of different materials, and 
revolve all around ${ }^{[4]}$. China is a great country of dressing. Although our studies in this field started late, China develops fast. At present, the development of 3D virtual fitting system begins in Hong Kong, Shenzhen, and Beijing. Some APPs have entered into commercialization phase, which have favorable development prospect.

However, there is still huge study space; there are still many difficulties to be solved; we still make great efforts, if 3D virtual fitting system can be realized actually. At present, virtual fitting system can reflect color, style, and matching, but it fails to reflect whether the fabric of real products is consistent with its description online and whether the dressing is well fitted. There are many technical difficulties in practical application, such as how to improve the accuracy of 3D human body measurement and model, how to solve the problem that data storage needs strong computer allocation, how to reflect color, lustre, and fabric of dressing on the screen accurately, how to realize highly real physical collision effect, and how to reduce total cost and so on.

Virtual fitting system brings new concept and new mode to human-computer interaction; makes content be more reasonable and harmonious. The application of virtual fitting technology has been new motive power that promotes mobile e-commerce development in clothing industry; changes people's lifestyle; promotes technological progress and social revolution. In the near future, it is predictable that, with the further development of smart environment, 3D imaging, 3D outdoor scene, and multi-sensory interaction technology, virtual fitting design will be simpler and more humanized. Operation will be more convenient and faster. System will be more natural and interesting. Development will be standard, smart, networking, and highly simulated. Virtual fitting technology has important application value to investors in clothing industry, which will welcome a new and fast development period with wider prospect and trend.

\section{Conclusion}

In conclusion, with the improvement of technology, the development of computer technology, and scientific researchers' improvement to virtual system technology, a high-tech 3D virtual fitting system will be reflected. Fresh, interesting, and practical virtual fitting system will make online clothing purchase market be more vigorous with irreplaceable position ${ }^{[5]}$. At that time, online shopping will be an indispensable part in people's shopping life gradually. Each user will have a virtual model, who can fit on new clothes in different online stores. 3D virtual fitting system will be generally accepted new mode for online sales and clothing purchase.

\section{Acknowledgement}

Non Doctoral Youth Development Fund Project Number: 201425Y

\section{References}

[1]. Zhou Chao, Xu Chaoyang, and Yu Haili, Study on 3D Virtual Fitting Problem in Clothing Purchase Online [J], Fashion Guide 2013: (1):41-45.

[2]. Zeng Yun, Chen Yingying, and Zhang Yue, Online Virtual Fitting System based on Human Body Identification [J], Television Technology, 2014: (11):206-210.

[3] .Zhang Chaoyang and Dou Junxia, Dress Design System based on 3D Interaction [J], Research and Exploration in Laboratory, 2014;(12):117-120.

[4]. Yang Chenhui and Chen Chen, Model Transformation in Virtual Fitting System [J], Journal of Xiamen University (Natural Science).

[5] .Cheng Yixuan, Discussion on Application of 3D Fitting Technology in Clothing Purchasing Online [J], Modern Business, 2011(18).35. 